\title{
Event-Triggered Consensus Control for Leader-Following Multiagent Systems Using Output Feedback
}

\author{
Yang Liu $\mathbb{D}^{1,2}$ and Xiaohui Hou ${ }^{1}$ \\ ${ }^{1}$ School of Automation Science and Electrical Engineering, Beihang University, Beijing 100191, China \\ ${ }^{2}$ The Seventh Research Division, Beihang University, Beijing 100191, China \\ Correspondence should be addressed to Yang Liu; ylbuaa@163.com
}

Received 8 May 2018; Accepted 10 July 2018; Published 15 August 2018

Academic Editor: Yimin Zhou

Copyright (C) 2018 Yang Liu and Xiaohui Hou. This is an open access article distributed under the Creative Commons Attribution License, which permits unrestricted use, distribution, and reproduction in any medium, provided the original work is properly cited.

\begin{abstract}
The event-triggered consensus control for leader-following multiagent systems subjected to external disturbances is investigated, by using the output feedback. In particular, a novel distributed event-triggered protocol is proposed by adopting dynamic observers to estimate the internal state information based on the measurable output signal. It is shown that under the developed observer-based event-triggered protocol, multiple agents will reach consensus with the desired disturbance attenuation ability and meanwhile exhibit no Zeno behaviors. Finally, a simulation is presented to verify the obtained results.
\end{abstract}

\section{Introduction}

Consensus is a basic problem in the cooperative control of multiagent systems [1-5], which is generally realized through the behavior-based method or the leader-following approach. To be specific, the leader-following consensus problem has been studied in [6-12] from different perspectives, where all the following agents can reach consensus by tracking a real or virtual leader based on local interactions. However, all the work mentioned above requires that the control system is implemented in a continuous or timesampled triggered way, which would consume some unnecessary energy and computing resources in applications.

To reduce the resource consumption, the event-triggered scheme has been applied to the consensus control problem. In particular, the event-triggered consensus of leaderfollowing multiagent systems was studied in [13-16], where the dynamics of agents was restricted to single- or doubleintegrator. Furthermore, the general linear multiagent system was considered in [17-21]. In [18], a distributed eventtriggered strategy was proposed with state-dependent threshold so that the following agents could asymptotically track the leader without continuous communication. But it was assumed that all the following agents were aware of state information of the leader. In [19], the distributed, centralized, and clustered event-triggered schemes were proposed for different network topologies, which could reduce the frequency of controller updates. In [21], an existing continuous control law was extended with a novel event-triggered condition, and it was proved that the system remained the desired performance with much lower controller updating frequency. In the literatures mentioned above, the consensus protocols and event-triggered conditions are both designed using the internal state information that is very hard or even impossible to be accurately obtained in real systems [22]. In addition, the external disturbance always exists in realistic situations, whose influence also has to be taken into account.

In this paper, the output-feedback event-triggered consensus is investigated for leader-following multiagent systems with high-order linear dynamics, subject to external disturbances. A novel distributed control protocol is proposed with an observer form, by using the local output information. Then, sufficient conditions are derived to guarantee that the system can reach consensus asymptotically with the desired disturbance attenuation ability but without Zeno behavior. The contributions of our research are as follows. The difficulties in directly obtaining full states is overcome by designing a local state observer, whose output is used to 
generate the event-triggered consensus protocol. Besides, in the developed scheme, the consensus algorithm, the state observer, and the event-triggering monitor are all implemented in a distributed and asynchronous way, which is applicable in practice and can reduce the controller updating frequency.

The rest of the paper is arranged as follows. Section 2 gives the preliminaries on graph theory and the problem formulation. In Section 3, the event-triggered scheme is proposed with a state observer, in which the internal states of each agent are estimated by using its output information. And it is proved that the desired consensus performance can be realized with no Zeno behavior. A simulation is given in Section 4, and Section 5 concludes the whole paper.

\section{Preliminaries and Problem Formulation}

2.1. Preliminaries. In a leader-following multiagent system, the communication topology among following agents is described by a graph $\mathscr{G}=(\mathscr{V}, \mathscr{E}, \mathscr{A})$. Assume that there are $n$ agents and let $\mathcal{N}=\{1, \ldots, n\}$. Then, $\mathscr{V}=\left\{v_{1}, v_{2}, \ldots, v_{n}\right\}$ denotes the set of nodes with node $v_{i}$ standing for the ith agent, and $\mathscr{E} \subseteq \mathscr{V} \times \mathscr{V}$ denotes the set of edges, in which the edge $\left(v_{j}, v_{i}\right)$ means information is transferred from agents $j$ to $i$. Besides, if $\left(v_{j}, v_{i}\right) \in \mathscr{E}$, node $v_{j}$ is called a neighbor of $v_{i} . \mathscr{A}=\left[a_{i j}\right]$ is named the adjacency matrix with $a_{i j} \geq 0$, where the positive element $a_{i j}$ is the weighing factor of edge $\left(v_{j}, v_{i}\right)$. On this basis, the Laplacian matrix of $\mathscr{G}$ can be defined as $\mathscr{L}=\mathscr{D}-\mathscr{A}$, in which $\mathscr{D}==\operatorname{diag}\left\{d_{1}, \ldots, d_{n}\right\}$ is called the degree matrix of $\mathscr{G}$ with $d_{i}=\sum_{j=1}^{n} a_{i j}$.

On the other hand, for the leading agent represented by node $v_{0}$, the edge $\left(v_{0}, v_{i}\right) /\left(v_{i}, v_{0}\right)$ represents that the information is exchanged between the $i$ th agent and the leader, whose weighing factor is denoted by $a_{0 i}=a_{i 0}$. In other words, the edges between following agents and the leader are bidirectional. Note that there is no control input for the leader, and the information from its neighboring agents is just used to determine the triggering time. To summarize, let $\overline{\mathscr{E}}$ be the edge set related to $n$ agents and one leader, then the set of neighbors of node $v_{i}$ is $\mathscr{N}_{i}=\left\{v_{j} \in\left\{\mathscr{V} \cup v_{0}\right\} \backslash\left(v_{j}, v_{i}\right) \in \overline{\mathscr{E}}\right\}$. The interaction matrix of the leader-following system is defined as $H=L+\Lambda$, where $\Lambda=\operatorname{diag}\left\{a_{10}, \ldots, a_{n 0}\right\}$.

To realize the consensus control of leader-following multiagent systems, the assumption on interaction graphs is made as follows [7].

Assumption 1. It is supposed that the interaction graph of the leader-follower system with directed communication has a spanning tree with the leader as root, or at least one agent in each connected component of $\mathscr{G}$ is connected to the leader for the undirected case.

2.2. Problem Formulation. Consider a leader-following multiagent system with $n$ followers and one leader. The $i$ th follower is modeled by a linear dynamic system with external disturbances:

$$
\begin{aligned}
& \dot{x}_{i}(t)=A x_{i}(t)+B u_{i}(t)+B_{1} \omega_{i}(t), \\
& y_{i}(t)=C x_{i}(t), \quad i=1,2, \ldots, n,
\end{aligned}
$$

where $x_{i}(t) \in \mathbb{R}^{m}$ is the state, $u_{i}(t) \in \mathbb{R}^{m_{1}}$ is the control input, $y_{i}(t) \in \mathbb{R}^{p}$ is the measuring output, and $\omega_{i}(t) \in \mathbb{R}^{m_{2}}$ is the external disturbance satisfying $\omega_{i}(t) \in L_{2}[0, \infty)$. The dynamics of the leader is

$$
\begin{aligned}
& \dot{x}_{0}(t)=A x_{0}(t), \\
& y_{0}(t)=C x_{0}(t),
\end{aligned}
$$

where $x_{0}(t) \in \mathbb{R}^{m}$ and $y_{0}(t) \in \mathbb{R}^{p}$ denote the state and output of the leader, respectively. Without loss of generality, it is assumed that $(A, B)$ is stabilizable, $(A, C)$ is observable, and $C$ is of full row rank.

Protocol $u_{i}(t)$ is said to solve the consensus problem if and only if the following equality is satisfied:

$$
\lim _{t \rightarrow \infty}\left(x_{i}(t)-x_{0}(t)\right)=0, \quad \forall i \in \mathcal{N} .
$$

However, the accurate consensus is hard to reach when there exist external disturbances. So the following controlled output is defined to measure the disagreement of agent $i$ to the leader agent:

$$
z_{i}(t)=x_{i}(t)-x_{0}(t), \quad i=1, \ldots, n .
$$

Let

$$
\begin{aligned}
& \omega(t)=\left[\begin{array}{lll}
\omega_{1}^{T}(t) & \cdots & \omega_{n}^{T}(t)
\end{array}\right]^{T} \in \mathbb{R}^{m_{2} n}, \\
& z(t)=\left[\begin{array}{lll}
z_{1}^{T} & \cdots & z_{n}^{T}(t)
\end{array}\right]^{T} \in \mathbb{R}^{m n},
\end{aligned}
$$

Obviously, if $z(t)=0$, then $z_{i}(t)=0$ for any $i \in \mathcal{N}$ and equivalently (3) holds. Therefore, the $H_{\infty}$ norm of the transfer function matrix from $\omega(t)$ to $z(t)$, denoted by $T_{z \omega}(s)$, can quantitatively measure the attenuation ability of the multiagent system against external disturbances. Combining with the definition of $H_{\infty}$ norm, the control objective is to make

$$
\left\|T_{z \omega}(s)\right\|_{\infty}=\sup _{v \in \mathbb{R}} \bar{\sigma}\left(T_{z \omega}(j v)\right)=\sup _{0 \neq \omega(t) \in L_{2}[0, \infty)} \frac{\|z(t)\|_{2}}{\|\omega(t)\|_{2}}<\gamma
$$

satisfied, which is equivalent to

$$
\int_{0}^{\infty}\|z(t)\|^{2} d t<\gamma^{2} \int_{0}^{\infty}\|\omega(t)\|^{2} d t, \forall \omega \in L_{2}[0, \infty),
$$

where $\gamma>0$ is the given $H_{\infty}$ performance index [23]. When (7) is satisfied for the closed-loop system, we say consensus is realized with the disturbance attenuation ability $\gamma$.

\section{Protocol Design and Consensus Analysis}

3.1. Output-Feedback Event-Triggered Protocol. To realize the consensus control using output information, state observers are first designed for the following agents and the leader as 


$$
\begin{aligned}
& \dot{\tilde{x}}_{i}(t)=A \tilde{x}_{i}(t)+B u_{i}(t)+G\left(\tilde{y}_{i}(t)-y_{i}(t)\right), \\
& \tilde{y}_{i}(t)=C \tilde{x}_{i}(t),
\end{aligned}
$$

and

$$
\begin{aligned}
& \dot{\tilde{x}}_{0}(t)=A \tilde{x}_{0}(t)+G\left(\tilde{y}_{0}(t)-y_{0}(t)\right), \\
& \tilde{y}_{0}(t)=C \tilde{x}_{0}(t),
\end{aligned}
$$

in which $\tilde{x}_{i}(t) \in R^{m}$ and $\tilde{x}_{0}(t) \in R^{m}$ are, respectively, the estimated states of the $i$ th agent and the leader. Combining with observers (8) and (9), the event-triggered protocol is developed as

$$
u_{i}(t)=K \sum_{j \in N_{i}} a_{i j}\left(e^{A\left(t-t_{k}^{i}\right)} \tilde{x}_{i}\left(t_{k}^{i}\right)-e^{A\left(t-t_{k}^{j}\right)} \tilde{x}_{j}\left(t_{k}^{j}\right)\right),
$$

where $t_{k}^{i}$ denotes the $k$ th triggering time of the $i$ th agent determined by the event-triggered condition

$$
\left\|e_{i}(t)\right\|=\sqrt{\sigma}\left\|\sum_{j \in N_{i}} a_{i j}\left(\tilde{x}_{i}(t)-\tilde{x}_{j}(t)\right)\right\|,
$$

with $e_{i}(t)=e^{A\left(t-t_{k}^{i}\right)} \tilde{x}_{i}\left(t_{k}^{i}\right)-\tilde{x}_{i}(t), \quad t \in\left[t_{k}^{i}, t_{k+1}^{i}\right)$.

Remark 1. Totally, (8), (9), (10), and (11) form the outputfeedback event-triggered protocol, which is implemented in a distributed and asynchronous way. Notice that there is an item $e^{A t}$ in the definition of $e_{i}(t)$ that can be regarded as a predictive factor. By this, the trigger frequency can be reduced, and the redundant triggers after achieving consensus can be also avoided. Similarly, to decrease the effect of sampling error to the control effect, the predictive factor is also used in the designed protocol $u_{i}(t)$.

3.2. Model Transformation. For analyzing the consensus performance, the nonzero consensus trajectory of a closedloop multiagent system is firstly converted into the origin by model transformations. Define the observing error as $h_{i}(t)=$ $\tilde{x}_{i}(t)-x_{i}(t), i=0, \ldots, n$, and let $\bar{x}_{i}(t)=\tilde{x}_{i}(t)-\tilde{x}_{0}(t), \bar{h}_{i}(t)=$ $h_{i}(t)-h_{0}(t)$, and $\bar{e}_{i}(t)=e_{i}(t)-e_{0}(t)$. By (8), (9), and (10), it is derived that

$$
\begin{aligned}
\dot{\bar{x}}_{i}(t) & =\dot{\tilde{x}}_{i}(t)-\dot{\tilde{x}}_{0}(t) \\
& =A \tilde{x}_{i}(t)+B u_{i}(t)+G C h_{i}(t)-A \tilde{x}_{0}(t)-G C h_{0}(t) \\
& =A\left(\tilde{x}_{i}(t)-\tilde{x}_{0}(t)\right)+B u_{i}(t)+G C\left(h_{i}(t)-h_{0}(t)\right) \\
& =A \bar{x}_{i}(t)+B u_{i}(t)+G C \bar{h}_{i}(t), \\
\dot{\bar{h}}_{i}(t) & =\dot{h}_{i}(t)-\dot{h}_{0}(t) \\
& =(A+G C) h_{i}(t)-B_{1} \omega_{i}(t)-(A+G C) h_{0}(t) \\
& =(A+G C) \bar{h}_{i}(t)-B_{1} \omega_{i}(t),
\end{aligned}
$$

and

$$
\begin{gathered}
u_{i}(t)=K \sum_{j \in N_{i}} a_{i j}\left[\left(e_{i}(t)+\tilde{x}_{i}(t)\right)-\left(e_{j}(t)+\tilde{x}_{j}(t)\right)\right] \\
=K \sum_{j \in N_{i}, j \neq 0} a_{i j}\left[\left(\bar{e}_{i}(t)+\bar{x}_{i}(t)\right)-\left(\bar{e}_{j}(t)+\bar{x}_{j}(t)\right)\right] \\
+K a_{i 0}\left[\bar{e}_{i}(t)+\bar{x}_{i}(t)\right] .
\end{gathered}
$$

Furthermore, by letting

$$
\begin{aligned}
& \bar{x}(t)=\left[\begin{array}{lll}
\bar{x}_{1}^{T}(t) & \cdots & \bar{x}_{n}^{T}(t)
\end{array}\right]^{T} \in \mathbb{R}^{m n}, \\
& u(t)=\left[\begin{array}{lll}
u_{1}^{T}(t) & \cdots & u_{n}^{T}(t)
\end{array}\right]^{T} \in \mathbb{R}^{m_{1} n}, \\
& \bar{y}(t)=\left[\begin{array}{lll}
\bar{y}_{1}^{T}(t) & \cdots & \bar{y}_{n}^{T}(t)
\end{array}\right]^{T} \in \mathbb{R}^{p n}, \\
& \bar{h}(t)=\left[\begin{array}{lll}
\bar{h}_{1}^{T}(t) & \cdots & \bar{h}_{n}^{T}(t)
\end{array}\right]^{T} \in \mathbb{R}^{m n}, \\
& \bar{e}(t)=\left[\begin{array}{lll}
\bar{e}_{1}^{T}(t) & \cdots & \bar{e}_{n}^{T}(t)
\end{array}\right]^{T} \in \mathbb{R}^{m n},
\end{aligned}
$$

we have

$$
u(t)=(H \otimes K)(\bar{e}(t)+\bar{x}(t))
$$

and then

$\dot{\bar{x}}(t)=\left(I_{n} \otimes A+H \otimes B K\right) \bar{x}(t)+(H \otimes B K) \bar{e}(t)+\left(I_{n} \otimes G C\right) \bar{h}(t)$,

$\dot{\bar{h}}(t)=\left[I_{n} \otimes(A+G C)\right] \bar{h}(t)-\left(I_{n} \otimes B_{1}\right) \omega(t)$.

$$
\text { Let } \bar{\xi}^{T}(t)=\left[\begin{array}{ll}
\bar{x}^{T}(t) & \bar{h}^{T}(t)
\end{array}\right]^{T} \text { and } \bar{\varepsilon}^{T}(t)=\left[\begin{array}{ll}
\bar{e}^{T}(t) & 0_{1 \times m n}
\end{array}\right]^{T} \text {, }
$$
then (16) and (4) can be rewritten as

$$
\begin{aligned}
& \dot{\bar{\xi}}(t)=\bar{A} \bar{\xi}(t)+\bar{E} \overline{\mathcal{\varepsilon}}(t)+\bar{W} \omega(t), \\
& z(t)=\bar{C} \bar{\xi}(t),
\end{aligned}
$$

where

$$
\begin{aligned}
& \bar{A}=\left[\begin{array}{cc}
I_{n} \otimes A+H \otimes B K & I_{n} \otimes G C \\
0 & I_{n} \otimes(A+G C)
\end{array}\right], \\
& \bar{E}=\left[\begin{array}{cc}
H \otimes B K & 0 \\
0 & 0
\end{array}\right], \\
& \bar{W}=\left[\begin{array}{cc}
0 & \\
-\left(I_{n} \otimes B_{1}\right)
\end{array}\right], \\
& \bar{C}=\left[\begin{array}{ll}
I_{n m} & -I_{n m}
\end{array}\right] .
\end{aligned}
$$

According to the definition equations of $\bar{x}_{i}(t)$ and $\bar{h}_{i}(t)$, if they are asymptotically stable at the origin, $\tilde{x}_{i}(t)$ and $h_{i}(t)$ would asymptotically equal to $\tilde{x}_{0}(t)$ and $h_{0}(t)$, respectively. Then $x_{i}(t)$ can asymptotically reach $x_{0}(t)$ by $x_{i}(t)=\tilde{x}_{i}(t)-$ $h_{i}(t)$ and $x_{0}(t)=\tilde{x}_{0}(t)-h_{0}(t)$. Therefore, the consensus of leader-following multiagent systems (1) and (2) is reformulated as the asymptotical stability problem of system (17). In other words, if (17) is asymptotically stable at the origin, then the multiple agents reach consensus. Furthermore, the norm $\left\|T_{z \omega}(s)\right\|_{\infty}$ remains unchanged before and after the 
model transformation, and thus the $H_{\infty}$ performance of system (17) is equivalent to that of the multiagent system. In conclusion, if (17) is asymptotically stable with the disturbance attenuation performance $\left\|T_{z \omega}(s)\right\|_{\infty}<\gamma$, then the multiagent system can reach consensus with the disturbance attenuation level $\gamma$.

\subsection{Consensus Analysis}

Lemma 1. (Schur complement formula): Let $S$ be a symmetric matrix of the partitioned form $S=\left[S_{i j}\right]$ with $S_{11} \in \mathbb{R}^{r \times r}, S_{11} \in$ $\mathbb{R}^{r \times(n-r)}$, and $S_{22} \in \mathbb{R}^{(n-r) \times(n-r)}$. Then, $S<0$ if and only if

$$
S_{11}<0, S_{22}-S_{21} S_{11}^{-1} S_{12}<0,
$$

or equivalently

$$
S_{22}<0, S_{11}-S_{12} S_{22}^{-1} S_{21}<0
$$

Theorem 1. For a given index $\gamma>0$, system (17) is asymptotically stable with the disturbance attenuating performance $\left\|T_{z \omega}(s)\right\|_{\infty}<\gamma$, if there exists a positive definite matrix $\bar{P}$ and a positive parameter $\alpha$ so that the following inequality is satisfied

$$
\left[\begin{array}{ccc}
\bar{P} \bar{A}+\bar{A}^{T} \bar{P}+\bar{C}^{T} \bar{C}+2 \alpha \sigma\left(\bar{H}^{T} \bar{H}+\bar{\Lambda}^{T} \bar{\Lambda}\right) & \bar{P} \bar{E} & \bar{P} \bar{W} \\
\bar{E}^{T} \bar{P} & -\alpha I & 0 \\
\bar{W}^{T} \bar{P} & 0 & -\gamma^{2} I
\end{array}\right]<0,
$$

where $\bar{A}, \bar{E}, \bar{W}$, and $\bar{C}$ are defined in (18), $\bar{H}=\left[H \otimes I_{m}\right.$ $\left.0_{n m \times n m}\right]$, and $\bar{\Lambda}=\left[\begin{array}{ll}\left(\sqrt{n} \mathbf{1}_{n}^{T} \Lambda\right) \otimes I_{m} & 0_{1 \times n m}\end{array}\right]$. That is, under the event-triggered protocol (8), (9), (10), and (11), the leaderfollowing multiagent systems (1) and (2) can reach consensus with the disturbance attenuation ability $\gamma$.

Proof. Firstly, the asymptotical stability is demonstrated under the assumption $\omega(t)=0$. According to the eventtriggered condition (11), it is derived that

$$
\begin{aligned}
\|e(t)\| & \leq \sqrt{\sigma}\left\|\left(H \otimes I_{m}\right) \bar{x}(t)\right\|, \\
& \triangleq \sqrt{\sigma}\|\bar{H} \bar{\xi}(t)\|,
\end{aligned}
$$

with $e(t)=\left[\begin{array}{lll}e_{1}^{T}(t) & \cdots & e_{n}^{T}(t)\end{array}\right]^{T}$, and $\left\|e_{0}(t)\right\|=\sqrt{\sigma} \|\left(\mathbf{1}_{n}^{T} \Lambda\right.$ $\left.\otimes I_{m}\right) \bar{x}(t) \|$. From the definition of $\bar{\varepsilon}(t)$ and the equality $\bar{e}(t)$ $=e(t)-\mathbf{1}_{n} \otimes e_{0}(t),\|\bar{\varepsilon}(t)\|=\|\bar{e}(t)\| \leq\|e(t)\|+\sqrt{n}\left\|e_{0}(t)\right\|$ follows and then $\|\bar{\varepsilon}(t)\| \leq \sqrt{\sigma}(\|\bar{H} \bar{\xi}(t)\|+\|\bar{\Lambda} \bar{\xi}(t)\|)$ is obtained.

Define a Lyapunov function $V=\bar{\xi}^{T}(t) \bar{P} \bar{\xi}(t)$ with a positive definite matrix $\bar{P}$. The derivative of $V$ satisfies

$$
\begin{aligned}
\dot{V} & =\dot{\bar{\xi}}^{T}(t) \bar{P} \bar{\xi}(t)+\bar{\xi}^{T}(t) \bar{P} \dot{\bar{\xi}}(t) \\
& =(\bar{A} \bar{\xi}(t)+\bar{E} \bar{\varepsilon}(t))^{T} \bar{P} \bar{\xi}(t)+\bar{\xi}^{T}(t) \bar{P}(\bar{A} \bar{\xi}(t)+\bar{E} \overline{\bar{\varepsilon}}(t)) \\
& =\bar{\xi}^{T}(t)\left(\bar{A}^{T} \bar{P}+\bar{P} \bar{A}\right) \bar{\xi}(t)+2 \bar{\xi}^{T}(t) \bar{P} \bar{E} \bar{\varepsilon}(t) \\
& \leq \bar{\xi}^{T}(t)\left(\bar{A}^{T} \bar{P}+\bar{P} \bar{A}+\alpha^{-1} \bar{P} \bar{E} \bar{E}^{T} \bar{P}\right) \bar{\xi}(t)+\alpha \bar{\varepsilon}^{T}(t) \bar{\varepsilon}(t) \\
& \leq \bar{\xi}^{T}(t)\left[\bar{A}^{T} \bar{P}+\bar{P} \bar{A}+\alpha^{-1} \bar{P} \bar{E} \bar{E}^{T} \bar{P}+2 \alpha \sigma\left(\bar{H}^{T} \bar{H}+\bar{\Lambda}^{T} \bar{\Lambda}\right)\right] \bar{\xi}(t),
\end{aligned}
$$

Applying Lemma 1 to the inequality (21) indicates $\dot{V}<0$, and therefore, system (17) is asymptotically stable.

Secondly, consider the $H_{\infty}$ performance of system (17) with disturbance $\omega(t) \neq 0$. Define a cost function as follows:

$$
J_{T}=\int_{0}^{T}\|z(t)\|^{2} d t-\gamma^{2} \int_{0}^{T}\|\omega(t)\|^{2} d t
$$

Then, under the zero initial condition, the following result is derived:

$$
\begin{aligned}
& J_{T}=\int_{0}^{T}\left[z(t) z(t)-\gamma^{2} \omega(t) \omega(t)\right] d t \\
& =\int_{0}^{T}\left[z^{T}(t) z(t)-\gamma^{2} \omega(t) \omega(t)+\dot{V}(t)\right] d t-V(T) \\
& =\int_{0}^{T}\left[\bar{\xi}^{T}(t) \bar{C}^{T} \bar{C} \bar{\xi}(t)-\gamma^{2} \omega^{T}(t) \omega(t)+2 \bar{\xi}^{T}(t) \bar{P}\right. \\
& \cdot(\bar{A} \bar{\xi}(t)+\bar{E} \bar{\varepsilon}(t)+\bar{W} \omega(t))] d t-V(T) \\
& =\int_{0}^{T}\left[\begin{array}{c}
\bar{\xi}(t) \\
\bar{\varepsilon}(t) \\
\omega(t)
\end{array}\right]^{T}\left[\begin{array}{ccc}
\bar{P} \bar{A}+\bar{A}^{T} \bar{P}+\bar{C}^{T} \bar{C} & \bar{P} \bar{E} & \bar{P} \bar{W} \\
\bar{E}^{T} \bar{P} & 0 & 0 \\
\bar{W}^{T} \bar{P} & 0 & -\gamma^{2} I
\end{array}\right]\left[\begin{array}{c}
\bar{\xi}(t) \\
\bar{\varepsilon}(t) \\
\omega(t)
\end{array}\right] d t-V(T) \\
& =\int_{0}^{T}\left[\begin{array}{c}
\bar{\xi}(t) \\
\bar{\varepsilon}(t) \\
\omega(t)
\end{array}\right]^{T}\left[\begin{array}{ccc}
\bar{P} \bar{A}+\bar{A}^{T} \bar{P}+\bar{C}^{T} \bar{C} & \bar{P} \bar{E} & \bar{P} \bar{W} \\
\bar{E}^{T} \bar{P} & 0 & 0 \\
\bar{W}^{T} \bar{P} & 0 & -\gamma^{2} I
\end{array}\right]\left[\begin{array}{c}
\bar{\xi}(t) \\
\bar{\varepsilon}(t) \\
\omega(t)
\end{array}\right] \\
& \cdot d t+\alpha \bar{\varepsilon}^{T}(t) \bar{\varepsilon}(t)-\alpha \bar{\varepsilon}^{T}(t) \bar{\varepsilon}(t)-V(T) \\
& \leq \int_{0}^{T}\left[\begin{array}{c}
\bar{\xi}(t) \\
\bar{\varepsilon}(t) \\
\omega(t)
\end{array}\right]^{T}\left[\begin{array}{ccc}
\bar{P} \bar{A}+\bar{A}^{T} \bar{P}+\bar{C}^{T} \bar{C} & \bar{P} \bar{E} & \bar{P} \bar{W} \\
\bar{E}^{T} \bar{P} & 0 & 0 \\
\bar{W}^{T} \bar{P} & 0 & -\gamma^{2} I
\end{array}\right]\left[\begin{array}{c}
\bar{\xi}(t) \\
\bar{\varepsilon}(t) \\
\omega(t)
\end{array}\right] \\
& \cdot d t+2 \alpha \sigma \bar{\xi}^{T}(t)\left(\bar{H}^{T} \bar{H}+\bar{\Lambda}^{T} \bar{\Lambda}\right) \bar{\xi}(t)-\alpha \bar{\varepsilon}^{T}(t) \bar{\varepsilon}(t)-V(T) \\
& \leq \int_{0}^{T}\left[\begin{array}{c}
\bar{\xi}(t) \\
\bar{\varepsilon}(t) \\
\omega(t)
\end{array}\right]^{T}\left[\begin{array}{ccc}
\bar{P} \bar{A}+\bar{A}^{T} \bar{P}+\bar{C}^{T} \bar{C}+2 \alpha \sigma\left(\bar{H}^{T} \bar{H}+\bar{\Lambda}^{T} \bar{\Lambda}\right) & \bar{P} \bar{E} & \bar{P} \bar{W} \\
\bar{E}^{T} \bar{P} & -\alpha I & 0 \\
\bar{W}^{T} \bar{P} & 0 & -\gamma^{2} I
\end{array}\right] \\
& \cdot\left[\begin{array}{c}
\bar{\xi}(t) \\
\bar{\varepsilon}(t) \\
\omega(t)
\end{array}\right] d t-V(T) .
\end{aligned}
$$

According to the inequality condition (21), it is proved that 


$$
J_{T}+V(T)<0,
$$

from which it yields

$$
\int_{0}^{T}\|z(t)\|^{2} d t+V(T)<\gamma^{2} \int_{0}^{T}\|\omega(t)\|^{2} d t .
$$

Let $T \rightarrow \infty$, we get

$$
\int_{0}^{\infty}\|z(t)\|^{2} d t+V(T)<\gamma^{2} \int_{0}^{\infty}\|\omega(t)\|^{2} d t
$$

which is equivalent to $\left\|T_{z \omega}(s)\right\|_{\infty}<\gamma$. Combining with Section 3.2, it is demonstrated that the closed-loop multiagent system reaches consensus with the desired disturbance attenuation ability $\left\|T_{z \omega}(s)\right\|_{\infty}<\gamma$. This completes the proof.

Remark 2. Based on Theorem 1, the gain matrices in the proposed event-triggered protocol, that is, $K$ and $G$, can be determined by the following steps.

Firstly, applying Lemma 1 to (21) yields an equivalent inequality

$$
\left[\begin{array}{cc}
\bar{P} \bar{A}+\bar{A}^{T} \bar{P}+\bar{C}^{T} \bar{C}+2 \alpha \sigma\left(\bar{H}^{T} \bar{H}+\bar{\Lambda}^{T} \bar{\Lambda}\right)+\gamma^{-2} \bar{P} \bar{W} \bar{W}^{T} \bar{P} & \bar{P} \bar{E} \\
\bar{E}^{T} \bar{P} & -\alpha I
\end{array}\right]<0 .
$$

Then pre- and postmultiplying (29) with $\operatorname{diag}\left\{\bar{P}^{-1}, \bar{P}^{-1}\right\}$

leads to the following equivalent result

$$
\left[\begin{array}{cc}
\bar{A} \bar{P}^{-1}+\bar{P}^{-1} \bar{A}^{T}+\bar{P}^{-1} \bar{C}^{T} \bar{C} \bar{P}^{-1}+2 \alpha \sigma \bar{P}^{-1}\left(\bar{H}^{T} \bar{H}+\bar{\Lambda}^{T} \bar{\Lambda}\right) \bar{P}^{-1}+\gamma^{-2} \bar{W} \bar{W}^{T} & \bar{E} \bar{P}^{-1} \\
\bar{P}^{-1} \bar{E}^{T} & -\alpha \bar{P}^{-1} \bar{P}^{-1}
\end{array}\right]<0,
$$

which can be guaranteed by

$$
\left[\begin{array}{cc}
\bar{A} \bar{P}^{-1}+\bar{P}^{-1} \bar{A}^{T}+\bar{P}^{-1} \bar{C}^{T} \bar{C} \bar{P}^{-1}+2 \alpha \sigma \bar{P}^{-1}\left(\bar{H}^{T} \bar{H}+\bar{\Lambda}^{T} \bar{\Lambda}\right) \bar{P}^{-1}+\gamma^{-2} \bar{W} \bar{W}^{T} & \bar{E} \bar{P}^{-1} \\
\bar{P}^{-1} \bar{E}^{T} & \alpha\left(I-2 \bar{P}^{-1}\right)
\end{array}\right]<0,
$$

according to the inequality $-\bar{P}^{-1} \bar{P}^{-1} \leq I-2 \bar{P}^{-1}$. Again, by Lemma 1 , we get an equivalent inequality to (31) as

$\left[\begin{array}{ccccc}\bar{A} \bar{P}^{-1}+\bar{P}^{-1} \bar{A}^{T}+\gamma^{-2} \bar{W} \bar{W}^{T} & \bar{E} \bar{P}^{-1} & \bar{P}^{-1} \bar{H}^{T} & \bar{P}^{-1} \bar{\Lambda}^{T} & \bar{P}^{-1} \bar{C}^{T} \\ \bar{P}^{-1} \bar{E}^{T} & \alpha\left(I-2 \bar{P}^{-1}\right) & 0 & 0 & 0 \\ \bar{H} \bar{P}^{-1} & 0 & -0.5(\alpha \sigma)^{-1} I & 0 & 0 \\ \bar{\Lambda} \bar{P}^{-1} & 0 & 0 & -0.5(\alpha \sigma)^{-1} I & 0 \\ \bar{C} \bar{P}^{-1} & 0 & 0 & 0 & -I\end{array}\right]<0$.

To summarize, it is shown that if (32) holds, then the consensus condition (21) is satisfied. In order to calculate the gain matrices, let $\bar{P}^{-1}$ take the form

$$
\bar{P}^{-1}=I_{n} \otimes \operatorname{diag}\{P, P\},
$$

where $P$ is a positive definite matrix. Then, by substituting (18) and (33) into (32) and denoting 


$$
\begin{array}{r}
K P \triangleq Q, \\
G C P \triangleq R,
\end{array}
$$

we obtain a linear matrix inequality (LMI) in terms of matrix variables $P, Q$, and $R$. Once the above three matrices are figured out by using the LMI toolbox of Matlab, the feedback matrices can be determined as $K=Q P^{-1}$ and $G=R P^{-1} C_{R}^{-1}$, where $C_{R}^{-1}$ is right-inverse matrix of $C$.

Theorem 2. Under the event-triggered protocol (8), (9), (10), and (11), the leader-following multiagent system does not exhibit Zeno behavior.

Proof. Let $t_{k}^{i}$ be the current time of agent $i$. If $t_{k+1}^{i}-t_{k}^{i}$ is proved to be strictly positive, then there exists no Zeno behavior in the system. By the event-triggered condition (11), $\left\|e_{i}(t)\right\| \leq \sqrt{\sigma}\left\|\sum_{j \in N_{i}} a_{i j}\left(\tilde{x}_{i}(t)-\tilde{x}_{j}(t)\right)\right\|, t \in\left[t_{k}^{i}, t_{k+1}^{i}\right)$ holds. For the following agents, the derivative of $\left\|e_{i}(t)\right\|$ satisfies

$$
\begin{aligned}
\frac{d}{d t}\left\|e_{i}(t)\right\| & \leq\left\|\dot{e}_{i}(t)\right\|=\left\|A e^{A\left(t-t_{k}^{i}\right)} \tilde{x}_{i}\left(t_{k}^{i}\right)-\dot{\tilde{x}}_{i}(t)\right\| \\
& =\left\|A e_{i}(t)-B u_{i}(t)-G C h_{i}(t)\right\| \\
& \leq\|A\|\left\|e_{i}(t)\right\|+\|B\|\left\|u_{i}(t)\right\|+\|G C\|\left\|h_{i}(t)\right\| .
\end{aligned}
$$

From $\dot{h}_{i}(t)=(A+G C) h_{i}(t)-B_{1} \omega_{i}(t)$, we get

$$
\begin{aligned}
\frac{d}{d t}\left\|h_{i}(t)\right\| & \leq\left\|h_{i}(t)\right\| \leq\|A+G C\|\left\|h_{i}(t)\right\|+\left\|B_{1} \omega_{i}(t)\right\| \\
& \leq\|A+G C\|\left\|h_{i}(t)\right\|+w_{m i}
\end{aligned}
$$

where $w_{m i}=\max _{t \in\left[t_{k}^{i}, t_{k+1}^{i}\right]}\left\|B_{1} \omega_{i}(t)\right\|$. So, $\left\|h_{i}(t)\right\| \leq\|h\|_{m i}$ holds with

$$
\begin{aligned}
\|h\|_{m i}= & \frac{w_{m i}}{\|A+G C\|}\left(e^{\|A+G C\|\left(t_{k+1}^{i}-t_{k}^{i}\right)}-1\right) \\
& +\left\|h_{i}\left(t_{k}^{i}\right)\right\| e^{\|A+G C\|\left(t_{k+1}^{i}-t_{k}^{i}\right)} .
\end{aligned}
$$

Combining the expression of $u_{i}(t)$ with the property of continuous functions, there exists

$$
\|u\|_{m i}=\max _{t \in\left[t_{k}^{i}, t_{k+1}^{i}\right]}\left\|K \sum_{j \in N_{i}} a_{i j}\left(e^{A\left(t-t_{k}^{i}\right)} \tilde{x}_{i}\left(t_{k}^{i}\right)-e^{A\left(t-t_{k}^{j}\right)} \tilde{x}_{j}\left(t_{k}^{j}\right)\right)\right\|,
$$

to make $\left\|u_{i}(t)\right\| \leq\|u\|_{m i}$ hold. Thus, the derivative of $\left\|e_{i}(t)\right\|$ satisfies

$$
\frac{d}{d t}\left\|e_{i}(t)\right\| \leq\|A\|\left\|e_{i}(t)\right\|+\varphi_{k}^{i}
$$

in which $\varphi_{k}^{i}=\|G C\|\|h\|_{m i}+\left\|B_{1}\right\|\|u\|_{m i}$.

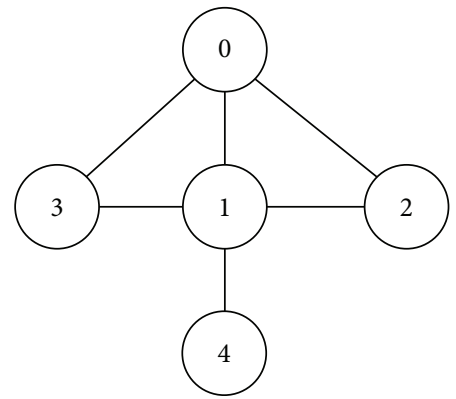

FIGURE 1: Communication topology of the leader-following system.

Similarly, for the leading agents, the derivative of $\left\|e_{0}(t)\right\|$ satisfies

$$
\begin{aligned}
\frac{d}{d t}\left\|e_{0}(t)\right\| \leq & \left\|\dot{e}_{0}(t)\right\|=\left\|A e^{A\left(t-t_{k}^{0}\right)} \tilde{x}_{0}\left(t_{k}^{0}\right)-\dot{\tilde{x}}_{0}(t)\right\| \\
= & \left\|A e_{0}(t)-G C h_{0}(t)\right\| \leq\|A\|\left\|e_{0}(t)\right\| \\
& +\|G C\|\left\|h_{0}(t)\right\|,
\end{aligned}
$$

which can be rewritten as

$$
\frac{d}{d t}\left\|e_{0}(t)\right\| \leq\|A\|\left\|e_{0}(t)\right\|+\varphi_{k}^{0}
$$

with $\varphi_{k}^{0}=\|G C\|\left\|h_{0}(t)\right\|$.

To summarize, for both leading and following agents, $(d / d t)\left\|e_{i}(t)\right\| \leq\|A\|\left\|e_{i}(t)\right\|+\varphi_{k}^{i}$ holds, and then it follows that

$$
\left\|e_{i}(t)\right\| \leq \frac{\varphi_{k}^{i}}{\|A\|}\left(e^{\|A\|\left(t-t_{k}^{i}\right)}-1\right), \quad t \in\left[t_{k}^{i}, t_{k+1}^{i}\right) .
$$

Before the consensus is achieved, $\| \sum_{j \in N_{i}} a_{i j}\left(\tilde{x}_{i}(t)-\tilde{x}_{j}(t)\right)$ $\|>0$ holds between any two triggering times, and there is a $\eta_{k}^{i}$ satisfying

$$
\sigma\left\|\sum_{j \in N_{i}} a_{i j}\left(\tilde{x}_{i}(t)-\tilde{x}_{j}(t)\right)\right\| \geq \eta_{k}^{i}>0, \quad t \in\left[t_{k}^{i}, t_{k+1}^{i}\right) .
$$

Obviously, there exists only one solution $t^{*}$ to $\left(\varphi_{k}^{i} /\|A\|\right)$ $\left(e^{\|A\|\left(t-t_{k}^{i}\right)}-1\right)=\eta_{k}$, which satisfies $t^{*}-t_{k}^{i}>0$ and $t^{*} \leq t_{k+1}^{i}$. Consequently, it is derived that $t_{k+1}-t_{k} \geq t^{*}-t_{k}^{i}>0$, which completes the proof.

\section{Simulation}

In this section, a numerical simulation is given to verify the theoretical results. A multiagent system consisting of one leader and four following agents is considered, 


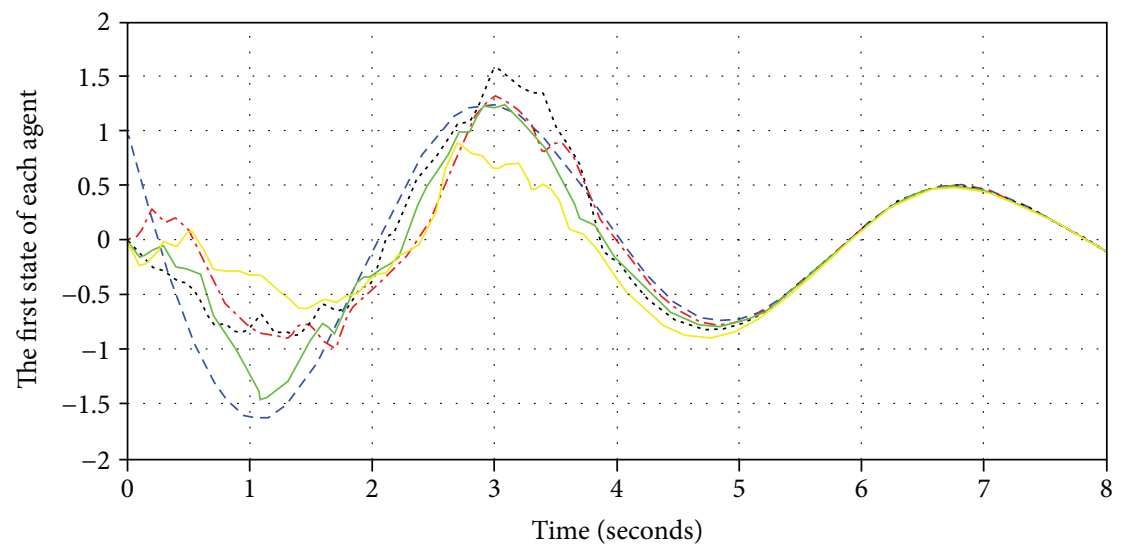

Figure 2: The first state component of each agent.

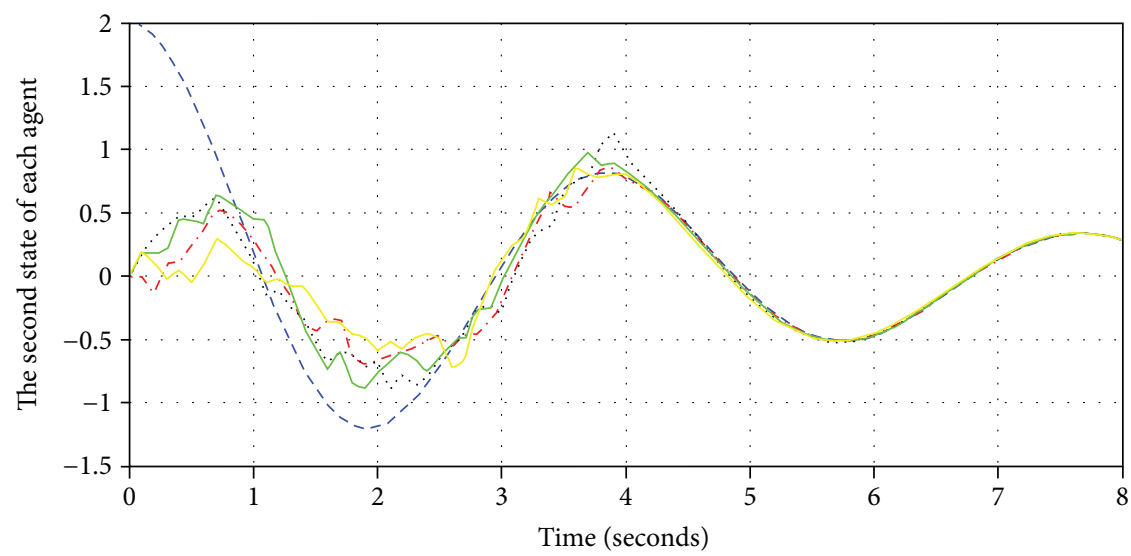

Figure 3: The second state component of each agent.

whose dynamics are modeled as (1) and (2) with system matrices

$$
\begin{aligned}
& A=\left[\begin{array}{ccc}
0 & -2 & 0 \\
1 & 0 & 0.5 \\
-0.5 & -2 & -1
\end{array}\right], \\
& B=B_{1}=\left[\begin{array}{ll}
1 & 0 \\
0 & 2 \\
0 & -1
\end{array}\right], \\
& C=\left[\begin{array}{lll}
0 & 1 & 0 \\
1 & 0 & 0 \\
0 & 0 & 1
\end{array}\right] .
\end{aligned}
$$

The external disturbances are simulated by white noises with brand limit $[0,0.1]$ and action period $[0,4 s]$. Set the initial states as $x_{0}=\left[\begin{array}{lll}1 & 2 & -2\end{array}\right]^{T}, x_{1}=x_{2}=x_{3}=x_{4}=\left[\begin{array}{lll}0 & 0 & 0\end{array}\right]^{T}$. The communication topology among agents is given by
Figure 1 with interaction factor $a_{i j}=0.2$. Besides, take $\alpha=5$, $\sigma=0.05$, and $\gamma=1$, and the gain matrices are determined as

$$
\begin{aligned}
& K=\left[\begin{array}{ccc}
-12.1723 & 0.9520 & 1.4748 \\
0.1182 & -4.3205 & 2.0867
\end{array}\right], \\
& G=\left[\begin{array}{ccc}
-0.0008 & -2.2198 & 0.2356 \\
-1.4996 & 0.0146 & 0.1125 \\
0.0366 & -0.2269 & -1.3914
\end{array}\right]
\end{aligned}
$$

The state trajectories of the multiagent system are shown in Figures 2-4, from which we can see that the following agents can reach consensus with the leader approximately during the first four seconds when there exist external disturbances and then achieve the exact consensus after the disappearance of disturbances. From Figure 5, it is obvious that the energy of the controlled output is lower than that of external disturbances, which indicates that the system realizes the $H_{\infty}$ disturbance attenuation performance with $\gamma=1$. Besides, the triggering times of each agent are $n_{0}=144, n_{1}=$ 


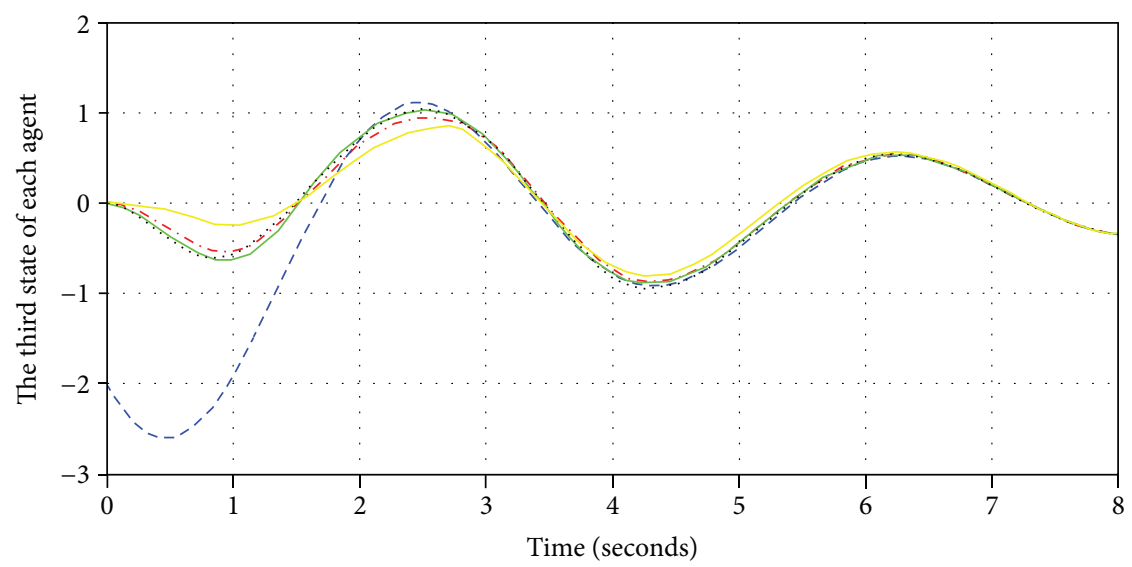

FIGURE 4: The third state component of each agent.

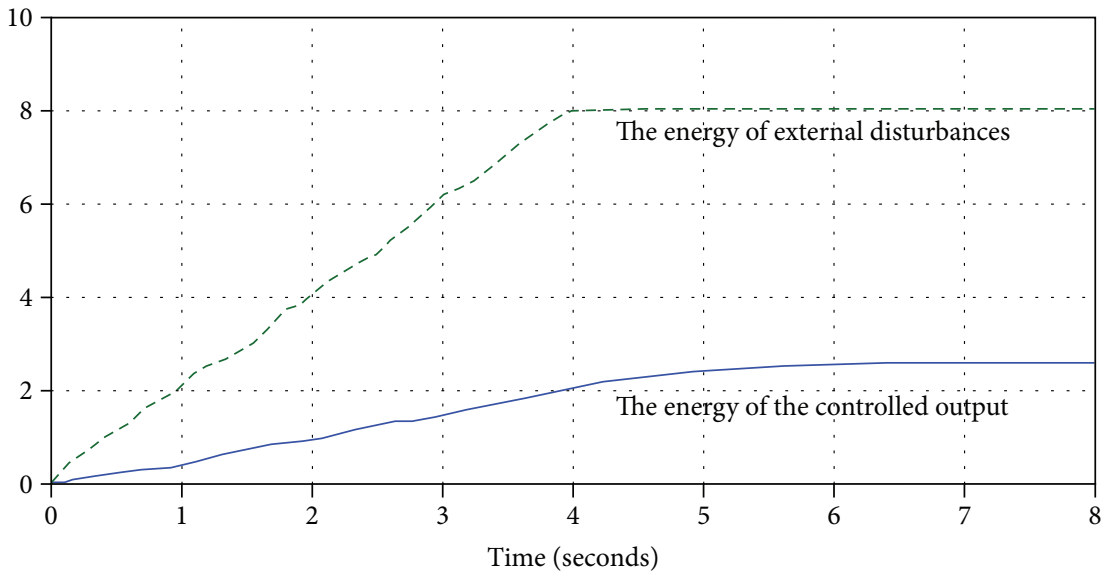

Figure 5: The energy of external disturbances and the controlled output.

223, $n_{2}=262, n_{3}=302$, and $n_{4}=354$, and no Zeno behavior occurs in the system.

\section{Conclusion}

In this paper, the output-feedback event-triggered consensus was addressed for the linear leader-following multiagent system with external disturbances. A distributed observer-based event-triggered protocol was proposed using the output information, under which the system could reach consensus with the desired disturbance attenuation ability and no Zeno behavior occurred. The effectiveness of the developed protocol was demonstrated by a numerical simulation.

\section{Data Availability}

In this paper, the simulation can be done by Matlab just using the model dynamics and proposed controller given in Section 4 , without any other data.

\section{Conflicts of Interest}

The authors declare that they have no conflicts of interest.

\section{Acknowledgments}

This work was supported by the National Natural Science Foundation of China (61473015, 61327807, 61520106010, and 61503231) and the Fundamental Research Funds for the Central Universities.

\section{References}

[1] B. Kaviarasan, R. Sakthivel, and S. Abbas, "Robust consensus of nonlinear multi-agent systems via reliable control with probabilistic time delay," Complexity, vol. 21, no. S2, 150 pages, 2016.

[2] Q. Chen, X. Ren, and J. Na, "Robust finite-time chaos synchronization of uncertain permanent magnet synchronous motors," ISA Transactions, vol. 58, pp. 262-269, 2015.

[3] Y. Liu and Y. Jia, "An iterative learning approach to formation control of multi-agent systems," Systems \& Control Letters, vol. 61, no. 1, pp. 148-154, 2012.

[4] P. Lin, Y. Jia, and L. Li, "Intelligent control in discrete time for autonomous systems," Discrete Dynamics in Nature \& Society, vol. 2016, pp. 1-13, 2016. 
[5] P. Lin, Y. Jia, and L. Li, "Distributed robust $H_{\infty}$ consensus control in directed networks of agents with time-delay," Systems \& Control Letters, vol. 57, no. 8, pp. 643-653, 2008.

[6] T. H. Lee, J. H. Park, D. H. Ji, and H. Y. Jung, "Leader-following consensus problem of heterogeneous multi-agent systems with nonlinear dynamics using fuzzy disturbance observer," Complexity, vol. 19, no. 4, 31 pages, 2014.

[7] Y. Liu and J. Lunze, "Leader-follower synchronisation of autonomous agents with external disturbances," International Journal of Control, vol. 87, no. 9, pp. 1914-1925, 2014.

[8] Y. Xu, Z. Zhen, and J. Chen, "Adaptive control for leaderfollowing consensus problem," in 2016 IEEE Chinese Guidance, Navigation and Control Conference (CGNCC), pp. 1654-1659, Nanjing, China, 2016.

[9] Z. Ma, Z. Liu, and Z. Chen, "Leader-following consensus for double-integrator dynamics with sampled-data," in 2016 35th Chinese Control Conference (CCC), pp. 7652-7657, Chengdu, China, 2016.

[10] Z. Zhao and Z. Lin, "Global leader-following consensus of a group of general linear systems using bounded controls," Automatica, vol. 68, pp. 294-304, 2016.

[11] Z. Zhang, Z. Zhang, and L. Li, "Dynamical sliding-mode control of leader-following multi-agent systems," in IECON 2017 - 43rd Annual Conference of the IEEE Industrial Electronics Society, pp. 6273-6278, Beijing, China, 2017.

[12] M. C. Fan and Y. Wu, "Global leader-following consensus of nonlinear multi-agent systems with unknown control directions and unknown external disturbances," Applied Mathematics and Computation, vol. 331, pp. 274-286, 2018.

[13] X. Y. Lou, B. Qi, and W. Feng, "Leader-following consensus of multiagent systems with event-triggered communication," in 2015 IEEE International Conference on Information and Automation, pp. 202-205, Lijiang, China, 2015.

[14] T. Xie, X. Liao, and H. Li, "Leader-following consensus in second-order multi-agent systems with input time delay: an event-triggered sampling approach," Neurocomputing, vol. 177, pp. 130-135, 2016.

[15] H. Li, X. Liao, T. Huang, and W. Zhu, "Event-triggering sampling based leader-following consensus in second-order multiagent systems," IEEE Transactions on Automatic Control, vol. 60, no. 7, pp. 1998-2003, 2015.

[16] N. Mu, X. Liao, and T. Huang, "Leader-following consensus in second-order multiagent systems via event-triggered control with nonperiodic sampled data," IEEE Transactions on Circuits and Systems II: Express Briefs, vol. 62, no. 10, pp. 1007-1011, 2015.

[17] X. Tan, J. Cao, and X. Li, "Consensus of leader-following multiagent systems: a distributed event-triggered impulsive control strategy," IEEE Transactions on Cybernetics, vol. PP, no. 99, pp. 1-10, 2018.

[18] N. Bekhadda, Q. H. Wu, and C. Du, "Distributed event triggered control with state dependent threshold for leader following consensus of general linear multi agent systems," in 2017 IEEE International Conference on Mechatronics and Automation (ICMA), pp. 1192-1197, Takamatsu, Japan, 2017.

[19] W. Xu, D. W. C. Ho, L. Li, and J. Cao, "Event-triggered schemes on leader-following consensus of general linear multiagent systems under different topologies," IEEE Transactions on Cybernetics, vol. 47, no. 1, pp. 212-223, 2017.
[20] W. Xu, D. W. C. Ho, L. Li, and J. Cao, "Leader-following consensus of general linear multi-agent systems: event-triggered schemes," in 2015 10th Asian Control Conference (ASCC), pp. 1-6, Kota Kinabalu, Malaysia, 2015.

[21] W. Hu, L. Liu, and G. Feng, "Leader-following consensus of linear multi-agent systems by distributed event-triggered control," in 2015 34th Chinese Control Conference (CCC), pp. 7050-7055, Hangzhou, China, 2015.

[22] Y. Jia, "Robust control with decoupling performance for steering and traction of $4 \mathrm{WS}$ vehicles under velocity-varying motion," IEEE Transactions on Control Systems Technology, vol. 8, no. 3, pp. 554-569, 2000.

[23] Y. Jia, "Alternative proofs for improved LMI representations for the analysis and the design of continuous-time systems with polytopic type uncertainty: a predictive approach," IEEE Transactions on Automatic Control, vol. 48, no. 8, pp. 14131416, 2003. 


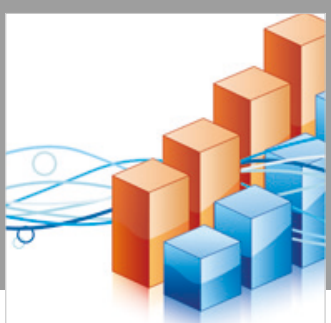

Advances in

Operations Research

\section{-n-m}
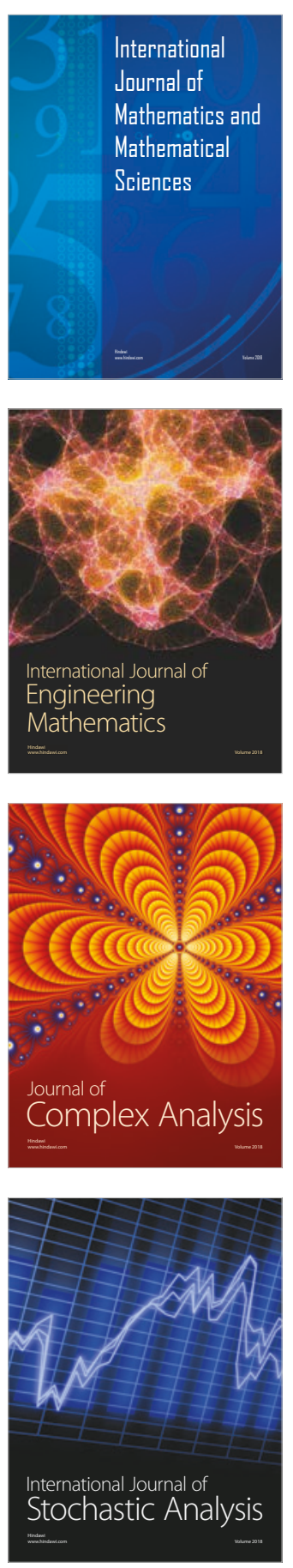
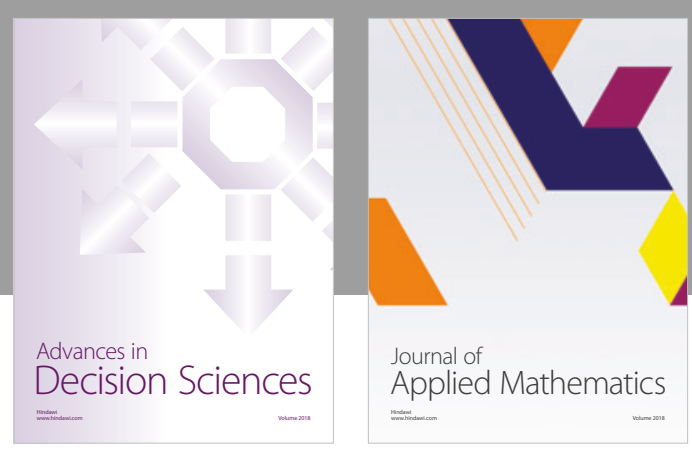

Journal of

Applied Mathematics
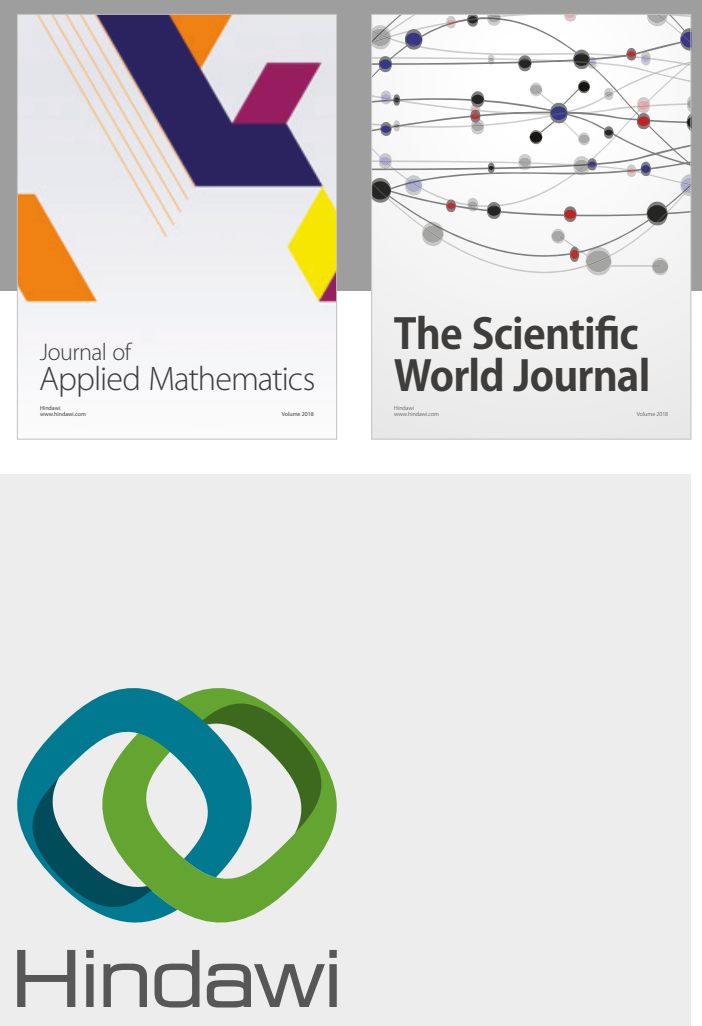

Submit your manuscripts at

www.hindawi.com

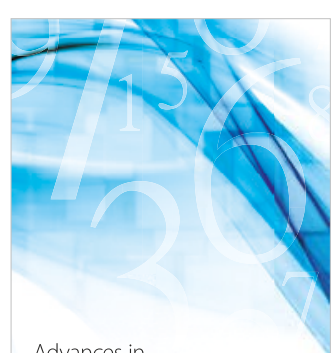

Advances in
Numerical Analysis
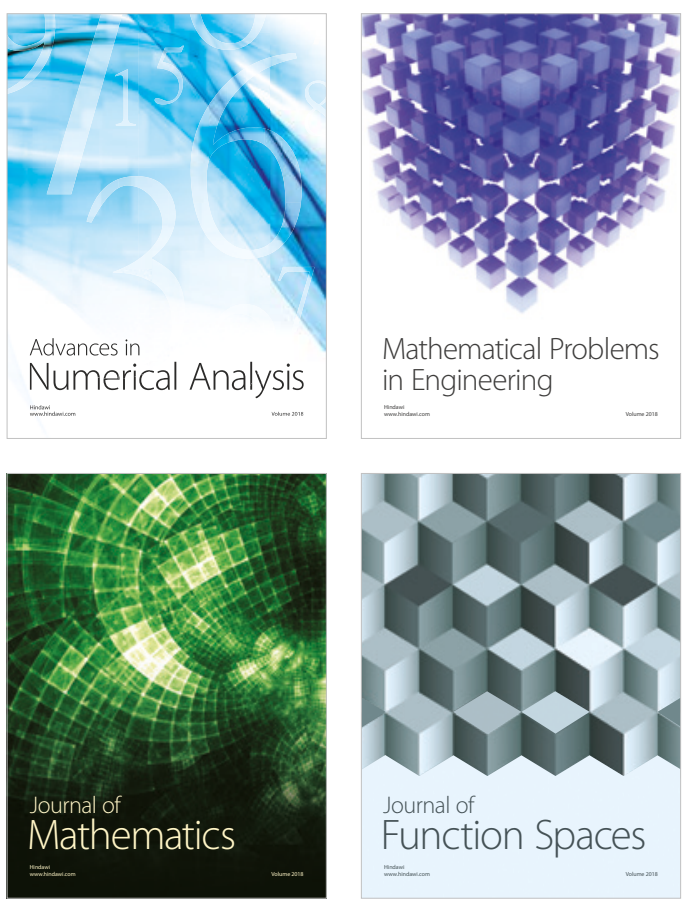

Mathematical Problems in Engineering

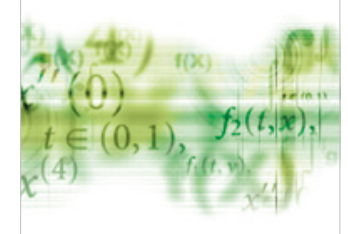

International Journal of

Differential Equations

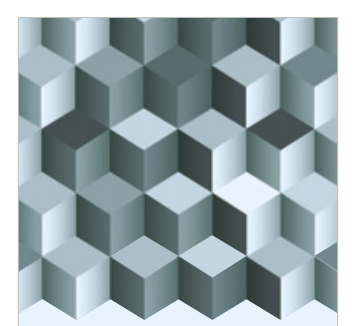

Journal of

Function Spaces
The Scientific

World Journal

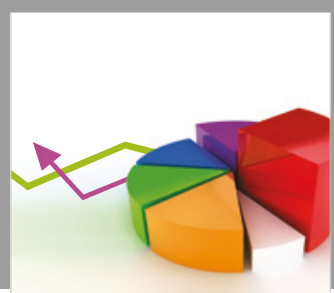

Journal of

Probability and Statistics
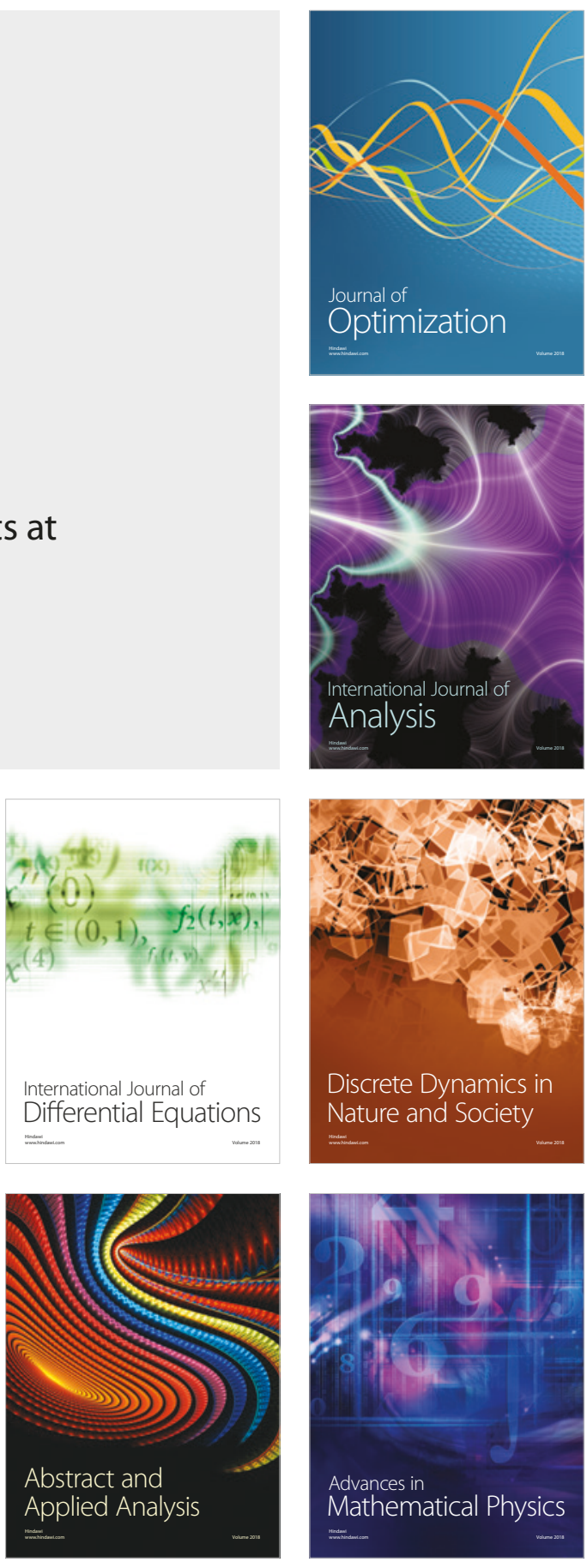